\title{
Benzyl and Sulfonyl Derivatives of N-(2,6-dimethylphenyl)-2- (piperazin-1-yl)acetamide (T2288): Biological Screening and Fingerprint applications
}

\author{
GHOUSE KHAN $^{1,2}$, SWAMY SREENIVASA ${ }^{1 *}$, SHIVARAJA GOVINDAIAH ${ }^{1}$ \\ and VIVEK CHANDRAMOHAN ${ }^{3}$
}

\author{
'Department of Studies and Research in Organic Chemistry, Tumkur University, Tumkur-572103, \\ Karnataka, India. \\ 2IDSG Government First Grade College, Chikkmagaluru-577101, Karnataka, India. \\ ${ }^{3}$ Department of Biotechnology, Siddaganga Institute of Technology, Tumkur-572103, \\ Karnataka, India. \\ *Corresponding author E-mail: drsreenivasa@yahoo.co.in \\ http://dx.doi.org/10.13005/ojc/350117
}

(Received: September 22, 2018; Accepted: December 29, 2018)

\section{ABSTRACT}

\begin{abstract}
A series of five N-(2,6-dimethylphenyl)-2-(piperazin-1-yl)acetamide (T2288) sulfonamides 6a-e and its five alkylated piperazine derivatives 8 a-e have been synthesized, characterized and screened for antibacterial, antifungal and anthelmintic activity. Some of the compounds showed significant biological activities. Molecular docking to crystal structures of target proteins revealed that, active compounds show similar binding poses as that of standards, indicating good correlation of the binding energy with observed in vitro data for the active compounds. Finally, the study of latent fingerprint analysis showed that the compound $6 \mathrm{c}$ exhibits good stickiness and finger rhythm without dense dust. The resulting compound can be used to detect fingerprints on all types of flat surfaces and hence easily accepted for detecting hidden fingerprints. This research can offer an excellent setting that can lead to the discovery of potential antibacterial, antifungal, anthelmintic and fingerprint agents.
\end{abstract}

Keywords: T2288, Sulphonamide, Molecular Docking, Piperazine, Finger Print, Biological screening.

\section{INTRODUCTION}

In recent years, the situation of antimicrobial resistance to a number of available antimicrobial agents is increasing drastically. According to World Health Organization report, from different parts of the world, indicates an overall decrease in the antibiotic effectiveness on global surveillance of antimicrobial resistance ${ }^{1}$. Infections caused by most of the microorganisms including Staphylococcus aureus, Escherichia coli, Proteus vulgaris, Pseudomonas aeruginosa, Salmonella typhi, Salmonella paratyphi and some fungi acquiring resistant day by day to the presently available antimicrobial agents and are the

This is an Open Access article licensed under a Creative Commons license: Attribution 4.0 International (CC- BY). Published by Oriental Scientific Publishing Company @ 2018 
most prevalent of fatal infectious diseases ${ }^{2-4}$. The problem is still very severe in case of Mycobacterium tuberculosis and hence needs a new potent antimicrobial drug with new mechanism of action ${ }^{5}$. In this context, chemical modification of approved drug molecules is a well-known approach affording the new chemical entities (NCE) with enhanced therapeutic characteristics. In this view, Piperazine derivatives emerged as good candidates as they showed broad spectrum of pharmacological activities like antidepressant ${ }^{6}$, anticancer ${ }^{7}$, antibacterial ${ }^{8}$, anthelmintic ${ }^{9}$, antimycobacterial ${ }^{10}$, antifungal ${ }^{11}$, anticonvulsant ${ }^{12}$ and antimalarial ${ }^{13}$. The presence of these heterocycles can be viewed in several wellknown drugs, like fluphenazine as antipsychotic ${ }^{14,15}$, cyclizine as antihistamine ${ }^{16}$, trimetazidine as antianginal ${ }^{17}$.

Many scientific reports reveals that sulfonyl group when linked to piperazine derivatives possess wide pharmacological activities like anti-inflammatory ${ }^{18}$, antidiabetic ${ }^{19}$, anticancer ${ }^{20-23}$, antibacterial ${ }^{24-25}$, antiprotozoal ${ }^{26}$, antifungal ${ }^{27-28}$, nonpeptidic vasopressin receptor antagonists ${ }^{29}$ and translation initiation inhibitors ${ }^{30-31}$. In addition, many acetamide derivatives have been reported to exhibit various biological activities such as anthelmintic, anticonvulsant, antioxidant, anti-inflammatory, anti-arthritic, anticancer, anti-bacterial and antifungal ${ }^{32-36}$. Some acetamide derivatives were found to be used in the development of enzymes that prevent the replication of HIV virus ${ }^{37,38}$. Certain acetamide compounds potentially inhibit the enzyme farnesyltransferase, a major strategy for the development of novel potential anticancer drugs $^{39}$. Some Arylacetamides reported as antimicrobial agents such as herbicides, antifungal and disinfectants ${ }^{40-41}$. A number of thioacetamide and sulfonyl acetamide derivatives of glycosyl were found to possess antitubercular activity ${ }^{42-44}$. These three active pharmacophores viz, piperazine, sulfonyl and acetamide groups, if they combined together would produce new molecular templates hope to exhibit enhanced biological activities. So we inspired and synthesized hybrid derivatives and test their biological activities.

\section{EXPERIMENTAL}
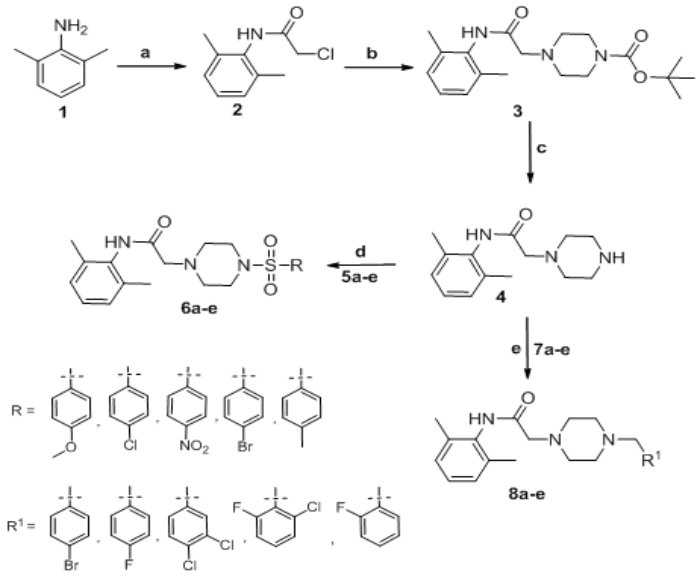

Scheme 1. Synthesis of Target compounds. Reagents and conditions: (a) 2-chloroacetyl chloride, $\mathrm{NaOH}, \mathrm{DCM}, 0^{\circ} \mathrm{C}$, 3 h; (b) N-Boc Piperazine, TEA, $\mathrm{CH}_{3} \mathrm{CN}, 2^{\circ} \mathrm{C}, 4$ h; (c) TFA, $\mathrm{DCM}, 0^{\circ} \mathrm{C}, 3 \mathrm{~h}$; (d) $\mathrm{RSO}_{2} \mathrm{Cl} 5 \mathrm{a}-\mathrm{e}, \mathrm{DCM}, \mathrm{TEA}, 25^{\circ} \mathrm{C}, 8 \mathrm{~h}$; (e) $\mathrm{R}^{1} \mathrm{CH}_{2} \mathrm{Cl} 7 \mathrm{a}-\mathrm{e}, \mathrm{K}_{2} \mathrm{CO}_{3}$, MeCN, rt, 10 hours

\section{MATERIALS AND METHODS}

Melting points reported were determined by open capillary method. Structures were confirmed using infrared spectroscopy, nuclear magnetic spectroscopy like proton and carbon ${ }^{-13}$ finally by LC-MS data. FT-IR Spectra was recorded in Jasco FT-IR spectrometer, ${ }^{1} \mathrm{H}$ and ${ }^{13} \mathrm{C}$ NMR spectra were recorded at $399.65 \mathrm{MHz}$ and $100.40 \mathrm{MHz}$ using $\mathrm{CDCl}_{3}$. The chemical shift was reported in $\mathrm{ppm}$. The LC-MS were recorded using Waters Alliance 2795 separation module and Waters Micromass LCT mass detector. All chemicals were purchased from Merck India, Spectrochem and Sigma-Aldrich. Solvent and chemicals used are of AR grade. All bacterial strains were purchased from CSIR - National Chemical Laboratory (NCL), Pune, India.

\section{Synthesis of N-(2,6-dimethylphenyl)-2-(piperazin- 1-yl)acetamide (4)}

Intermediate 4 was synthesized as per the reported literature ${ }^{54}$. m.p $118^{\circ} \mathrm{C}$ and yield $72 \%$.

\section{Spectral characterization of $\mathrm{N}-(2,6-$ dimethylphenyl)- 2-(piperazin-1-yl)acetamide (4)}

${ }^{1} \mathrm{H}$ NMR $\left(400 \mathrm{MHz}, \mathrm{CDCl}_{3}, \mathrm{TMS}\right): \delta=8.64$ (s, $1 \mathrm{H}, \mathrm{NH}), 7.02-7.03(\mathrm{~m}, 3 \mathrm{H}, \mathrm{Ar}-\mathrm{H}), 3.08(\mathrm{~s}, 2 \mathrm{H}$, $\left.\mathrm{CH}_{2}\right)$, 2.84-2.87 (t, 4H, $\left.\mathrm{CH}_{2}\right), 2.56-2.57\left(\mathrm{t}, 4 \mathrm{H}, \mathrm{CH}_{2}\right)$, $2.16\left(\mathrm{~s}, 6 \mathrm{H}, \mathrm{CH}_{3}\right) ;{ }^{13} \mathrm{C} \mathrm{NMR}\left(100 \mathrm{MHz}, \mathrm{CDCl}_{3}, \mathrm{TMS}\right)$ : $\delta=168.39,134.89,133.73,128.75,126.99,76.92$, 62.38, 46.22, 18.55. Mass: $\mathrm{m} / \mathrm{z} 248.1$. 
General procedure for the synthesis of compounds 6a-e

An equimolar mixture of $\mathrm{N}$-(2,6-dimethy Iphenyl)-2-(piperazin-1-yl)acetamide 4 (0.001 mol) and different substituted benzene sulfonyl chlorides $5 a-e(0.001 \mathrm{~mol})$ in methylene dichloride were stirred for about $8 \mathrm{~h}$ in presence of triethylamine at $25^{\circ} \mathrm{C}$. The completion of the reaction was checked by TLC. Then it was poured into ice-cold water and extracted with MDC. The organic layer was washed with brine solution, dried over anhydrous sodium sulphate and concentrated under reduced pressure to get piperazine sulphonamide derivatives 6a-e in high yield.

\section{General procedure for the synthesis of compounds 8a-e}

To a solution of $\mathrm{N}$-(2,6-dimethylphenyl)-2(piperazin-1-yl)acetamide 4 in acetonitrile, different substituted benzyl chlorides $7 \mathrm{a}-\mathrm{e}(0.001 \mathrm{~mol})$ were slowly added drop wise in presence of $\mathrm{K}_{2} \mathrm{CO}_{3}(0.003$ mol). Reaction mixture was stirred for $10 \mathrm{~h}$ at $25^{\circ} \mathrm{C}$ and completion of reaction was checked by TLC. Reaction mixture was poured into ice-cold water and extracted with methylene dichloride. The organic layers was washed with brine solution, dried over anhydrous sodium sulphate and concentrated to get alkylated piperazine derivatives 8a-e in good yield.

\section{N-(2,6-dimethylphenyl)-2-(4-((4-methoxyphenyl) sulfonyl)piperazin-1-yl)acetamide 6a}

Colour: white solid, Yield: 91, m.p:180-181 ${ }^{\circ} \mathrm{C} .{ }^{1} \mathrm{H}$ NMR $\left(400 \mathrm{MHz}, \mathrm{CDCl}_{3}, \mathrm{TMS}\right): \delta=8.28$ (s, $1 \mathrm{H}, \mathrm{NH}), 7.68-7.66(\mathrm{t}, \mathrm{J}=8 \mathrm{~Hz}, 2 \mathrm{H}, \mathrm{Ar}-\mathrm{H}), 7.07-6.97$ (m, 5H, Ar-H), 3.84 (s, 3H, $\left.\mathrm{OCH}_{3}\right), 3.17$ (s, 2H, $\left.\mathrm{CH}_{2}\right), 3.06$ (s, 4H, $\left.\mathrm{CH}_{2}\right), 2.75-2.72(\mathrm{t}, \mathrm{J}=12 \mathrm{~Hz}, 4 \mathrm{H}$, $\left.\mathrm{CH}_{2}\right), 2.13\left(\mathrm{~s}, 6 \mathrm{H}, \mathrm{CH}_{3}\right) \cdot{ }^{13} \mathrm{C} \mathrm{NMR}\left(100 \mathrm{MHz}, \mathrm{CDCl}_{3}\right.$, TMS): $\delta=167.78,163.22,135.02,133.32,129.89$, 128.27, 127.37, 126.92, 114.34, 61.20, 55.62, 52.80, 46.06, 18.5. IR (KBr): $\delta=1662.2(\mathrm{CO}), 1322.2$ ( $\mathrm{S}=\mathrm{O}$ asymmetric), 1170.3 ( $\mathrm{S}=\mathrm{O}$ symmetric). LCMS m/z: cal. 417.17; found $418.25(\mathrm{M}+1)$.

\section{2-(4-((4-chlorophenyl)sulfonyl)piperazin-1-yl)-N- (2,6-dimethylphenyl)acetamide 6b}

Colour : white solid, Yield: 90, m.p:188-189 ${ }^{\circ} \mathrm{C} .{ }^{1} \mathrm{H}$ NMR $\left(400 \mathrm{MHz} \mathrm{CDCl}_{3}, \mathrm{TMS}\right): \delta=8.26(\mathrm{~s}, 1 \mathrm{H}$, $\mathrm{NH}), 7.68-7.66(\mathrm{~d}, \mathrm{~J}=8 \mathrm{~Hz}, 2 \mathrm{H}, \mathrm{Ar}-\mathrm{H}), 7.51-7.49$ (d, J = 8Hz, 2H, Ar-H), 7.07-7.02 (m, 3H, Ar-H), 3.18 (s, $2 \mathrm{H}, \mathrm{CH}_{2}$ ), $3.08\left(\mathrm{~s}, 4 \mathrm{H}, \mathrm{CH}_{2}\right), 2.76-2.73(\mathrm{t}, \mathrm{J}=12 \mathrm{~Hz}$, $\left.4 \mathrm{H}, \mathrm{CH}_{2}\right), 2.13\left(\mathrm{~s}, 6 \mathrm{H}, \mathrm{CH}_{3}\right) .{ }^{13} \mathrm{C} \mathrm{NMR}(100 \mathrm{MHz}$, $\left.\mathrm{CDCl}_{3}, \mathrm{TMS}\right): \delta=167.60,139.67,135.03,134.05$,
133.29, 129.12, 128.29, 127.405, 61.22, 52.77, 45.95, 18.56. IR (KBr): $\delta=1665.8$ (CO), 1321.4 ( $\mathrm{S}=\mathrm{O}$ asymmetric), 1169.3 ( $\mathrm{S}=\mathrm{O}$ symmetric). LCMS m/z: cal. 421.12; found 422.21(M+1).

N-(2,6-dimethylphenyl)-2-(4-((4-nitrophenyl) sulfonyl)piperazin-1-yl)acetamide 6c

Colour: white solid, Yield: 89, m.p:176-177

${ }^{\circ} \mathrm{C} .{ }^{1} \mathrm{H}$ NMR (400 MHz, $\left.\mathrm{CDCl}_{3}, \mathrm{TMS}\right): \delta=8.38-8.36$ (d, J = 8Hz, 2H; Ar-H), $8.21(\mathrm{~s}, 1 \mathrm{H}, \mathrm{NH}), 7.95-7.92$ (d, J = 12Hz, 2H, Ar-H), 7.07-7.02 (m, 3H, Ar-H), 3.22 (s, 2H, $\left.\mathrm{CH}_{2}\right), 3.16\left(\mathrm{~s}, 4 \mathrm{H}, \mathrm{CH}_{2}\right), 2.79\left(\mathrm{~s}, 4 \mathrm{H}, \mathrm{CH}_{2}\right)$, $2.12\left(\mathrm{~s}, 6 \mathrm{H}, \mathrm{CH}_{3}\right) \cdot{ }^{13} \mathrm{C} \mathrm{NMR}\left(100 \mathrm{MHz}, \mathrm{CDCl}_{3}, \mathrm{TMS}\right)$ : $\delta=167.35,150.36,141.7,134.14,133.18,128.87$, 127.47, 124.45, 61.19, 52.75, 45.95, 18.53. IR (KBr): $\delta=1659.4$ (CO), 1312.1( $\mathrm{S}=\mathrm{O}$ asymmetric), 1163.5 ( $\mathrm{S}=\mathrm{O}$ symmetric). LCMS m/z: cal. 432.15; found $433.24(\mathrm{M}+1)$.

2-(4-((4-bromophenyl)sulfonyl)piperazin-1-yl)-N(2,6-dimethylphenyl) acetamide 6d

Colour: white solid, Yield: 90, m.p: 184-185 ${ }^{\circ} \mathrm{C} .{ }^{1} \mathrm{H}$ NMR (400 MHz, $\left.\mathrm{CDCl}_{3}, \mathrm{TMS}\right): \delta=8.27(\mathrm{~s}, 1 \mathrm{H}$, $\mathrm{NH}), 7.65-7.63(\mathrm{~d}, \mathrm{~J}=8 \mathrm{~Hz}, 2 \mathrm{H}, \mathrm{Ar}-\mathrm{H}), 7.57-7.55$ (d, J = 8Hz, 2H, Ar-H), 7.05-7.00 (m, 3H, Ar-H), $3.15\left(\mathrm{~s}, 2 \mathrm{H}, \mathrm{CH}_{2}\right), 3.04\left(\mathrm{~s}, 4 \mathrm{H}, \mathrm{CH}_{2}\right), 2.71-2.69$ $\left(\mathrm{t}, \mathrm{J}=8 \mathrm{~Hz}, 4 \mathrm{H}, \mathrm{CH}_{2}\right), 2.11\left(\mathrm{~s}, 6 \mathrm{H}, \mathrm{CH}_{3}\right) \cdot{ }^{13} \mathrm{C} \mathrm{NMR}$ (100 MHz, $\left.\mathrm{CDCl}_{3}, \mathrm{TMS}\right): \delta=167.73,135.05,134.55$, 133.37, 132.50, 129.80, 128.26, 128.14, 127.36, $61.19,52.72,45.94,18.58 . \mathrm{IR}(\mathrm{KBr}): \delta=1650.0(\mathrm{CO})$, 1315.9 ( $\mathrm{S}=$ O asymmetric), 1159.7 ( $\mathrm{S}=$ O symmetric). LCMS m/z: cal. 465.07; found 468.16 (M+1).

\section{N-(2,6-dimethylphenyl)-2-(4-tosylpiperazin-1-yl) acetamide $6 \mathrm{e}$}

Colour: white solid, Yield: 87, m.p: 170-171 ${ }^{\circ} \mathrm{C} .{ }^{1} \mathrm{H}$ NMR (400 MHz, CDCl$\left.{ }_{3}, \mathrm{TMS}\right): \delta=8.29(\mathrm{~s}, 1 \mathrm{H}$, $\mathrm{NH}), 7.62-7.61(\mathrm{~d}, \mathrm{~J}=4 \mathrm{~Hz}, 2 \mathrm{H}, \operatorname{Ar}-\mathrm{H}), 7.32-7.31$ (d, J $=4 \mathrm{~Hz}, 2 \mathrm{H}, \mathrm{Ar}-\mathrm{H})$, 7.07-7.05 (m, 3H, Ar-H), 3.167-3.162 (d, J = 2Hz, 2H, $\mathrm{CH}_{2}$ ), $3.05\left(\mathrm{~s}, 4 \mathrm{H}, \mathrm{CH}_{2}\right)$, 2.73-2.72 (s, 4H, $\left.\mathrm{CH}_{2}\right), 2.12\left(\mathrm{~s}, 6 \mathrm{H}, \mathrm{CH}_{3}\right) \cdot{ }^{13} \mathrm{C} \mathrm{NMR}$ (100 MHz, $\left.\mathrm{CDCl}_{3}, \mathrm{TMS}\right): \delta=167.73,143.97,135.05$, 134.55, 133.37, 132.47, 129.20, 128.26, 127.77 , 127.36, 61.19, 52.72, 45.94, 18.58. IR (KBr): $\delta=$ 1649.1 ( $C=0), 1342.5$ ( $\mathrm{S}=\mathrm{O}$ asymmetric), 1140.1 ( $\mathrm{S}=$ O symmetric), 2918.89 (Ar-H). LCMS m/z: cal. 401.18; found $402.26(M+1)$.

2-(4-(4-bromobenzyl)piperazin-1-yl)-N-(2,6dimethylphenyl) acetamide $8 \mathrm{a}$

Colour: white solid, Yield: 92, m.p:130-131 $\mathrm{C}$. 
${ }^{1} \mathrm{H}$ NMR $\left(400 \mathrm{MHz}, \mathrm{CDCl}_{3}, \mathrm{TMS}\right): \delta=8.65(\mathrm{~s}, 1 \mathrm{H}$, $\mathrm{NH}), 7.43-7.41(\mathrm{~d}, \mathrm{~J}=8 \mathrm{~Hz}, 2 \mathrm{H}, \mathrm{Ar}-\mathrm{H}), 7.21-7.18$ (d, J = 12Hz, 2H, Ar-H), 7.08-7.04 (m, 3H, Ar-H), 3.47 (s, 2H, $\left.\mathrm{CH}_{2}\right), 3.17\left(\mathrm{~s}, 2 \mathrm{H}, \mathrm{CH}_{2}\right), 2.70\left(\mathrm{~s}, 4 \mathrm{H}, \mathrm{CH}_{2}\right), 2.52$ (s, $\left.4 \mathrm{H}, \mathrm{CH}_{2}\right), 2.19\left(\mathrm{~s}, 6 \mathrm{H}, \mathrm{CH}_{3}\right) \cdot{ }^{13} \mathrm{C} \mathrm{NMR}(100 \mathrm{MHz}$, $\left.\mathrm{CDCl}_{3}, \mathrm{TMS}\right): \delta=168.47,136.72,135.23,134.97$, 133.62, 132.66, 131.41, 130.76, 128.27, 127.18, 121.06, 61.58, 53.67, 18.62. IR (KBr): $\delta=1652.2(\mathrm{C}$ $=0)$. LCMS m/z: cal. 415.13 ; found $416.68(M+1)$.

\section{N-(2,6-dimethylphenyl)-2-(4-(4-fluorobenzyl)} piperazin-1-yl) acetamide $8 \mathrm{~b}$

Colour: white solid, Yield: 94, m.p:150-151 ${ }^{\circ} \mathrm{C} .{ }^{1} \mathrm{H} \mathrm{NMR}\left(400 \mathrm{MHz}, \mathrm{CDCl}_{3}, \mathrm{TMS}\right): \delta=8.66(\mathrm{~s}, 1 \mathrm{H}$, $\mathrm{NH}), 7.29-7.24(\mathrm{~d}, \mathrm{~J}=2 \mathrm{OHz}, 2 \mathrm{H}, \mathrm{Ar}-\mathrm{H}), 7.09-7.08$ (m, 3H, Ar-H), 7.02-6.97 (t, 2H, Ar-H), $3.48(\mathrm{~s}, 2 \mathrm{H}$, $\left.\mathrm{CH}_{2}\right), 3.19$ (s, 2H, $\left.\mathrm{CH}_{2}\right), 2.71\left(\mathrm{~s}, 4 \mathrm{H}, \mathrm{CH}_{2}\right), 2.51$ (s, $\left.4 \mathrm{H}, \mathrm{CH}_{2}\right), 2.21\left(\mathrm{~s}, 6 \mathrm{H}, \mathrm{CH}_{3}\right) \cdot{ }^{13} \mathrm{C} \mathrm{NMR}(100 \mathrm{MHz}$, $\left.\mathrm{CDCl}_{3}, \mathrm{TMS}\right): \delta=168.49,163.25,160.82,134.96$, $133.62,130.54,128.27,127.15,115.18,62.03$, $61.65,53.79,18.61 . \mathrm{IR}(\mathrm{KBr}): \delta=1648.2(\mathrm{C}=0)$. LCMS m/z: cal. 355.21; found $356.71(M+1)$.

\section{2-(4-(3,4-dichlorobenzyl)piperazin-1-yl)-N-(2,6- dimethylphenyl) acetamide 8c}

Colour: white solid, Yield: 93, m.p:142$143^{\circ} \mathrm{C} .{ }^{1} \mathrm{H}$ NMR $\left(400 \mathrm{MHz}, \mathrm{CDCl}_{3}, \mathrm{TMS}\right): \delta=8.64$ (s, $1 \mathrm{H}, \mathrm{NH}), 7.43-7.42(\mathrm{~d}, \mathrm{~J}=4 \mathrm{~Hz}, 1 \mathrm{H}, \mathrm{Ar}-\mathrm{H}), 7.38$ $7.36(\mathrm{~d}, \mathrm{~J}=8 \mathrm{~Hz}, 1 \mathrm{H}, \mathrm{Ar}-\mathrm{H}), 7.24$ (s, 1H, Ar-H), 7.17$7.14(\mathrm{~m}, 1 \mathrm{H}, \mathrm{Ar}-\mathrm{H}), 7.09-7.05(\mathrm{~m}, 3 \mathrm{H}, \mathrm{Ar}-\mathrm{H}), 3.46$ (s, $\left.2 \mathrm{H}, \mathrm{CH}_{2}\right), 3.19\left(\mathrm{~s}, 2 \mathrm{H}, \mathrm{CH}_{2}\right), 2.72\left(\mathrm{~s}, 4 \mathrm{H}, \mathrm{NCH}_{2}\right)$, $2.51\left(\mathrm{~s}, 4 \mathrm{H}, \mathrm{NCH}_{2}\right), 2.21\left(\mathrm{~s}, 6 \mathrm{H}, \mathrm{CH}_{3}\right) \cdot{ }^{13} \mathrm{C} \mathrm{NMR}(100$ $\left.\mathrm{MHz}, \mathrm{CDCl}_{3}, \mathrm{TMS}\right): \delta=168.41,138.48,134.96$, 133.60, 132.36, 131.03, 130.24, 128.28, 128.19, 127.17, 61.63, 53.14, 18.65; IR (KBr): $\delta=1639.4$ $(C=0)$. LCMS m/z: cal. 405.14; found $406.68(M+1)$.

\section{2-(4-(2-chloro-6-fluorobenzyl)piperazin-1-yl)-N- (2,6- dimethylphenyl) acetamide 8d}

Colour: white solid, Yield: 91, m.p:130-131 ${ }^{\circ} \mathrm{C} .{ }^{1} \mathrm{H}$ NMR $\left(400 \mathrm{MHz}, \mathrm{CDCl}_{3}, \mathrm{TMS}\right): \delta=8.67$ (s, $1 \mathrm{H}, \mathrm{NH})$, 7.24-7.18 (m, 2H, Ar-H), 7.09-7.07 (m, J $=2 \mathrm{~Hz}, 3 \mathrm{H}, \mathrm{Ar}-\mathrm{H}), 6.98(\mathrm{~m}, 1 \mathrm{H}, \mathrm{Ar}-\mathrm{H}), 3.72(\mathrm{~s}, 2 \mathrm{H}$, $\left.\mathrm{CH}_{2}\right), 3.17\left(\mathrm{~s}, 2 \mathrm{H}, \mathrm{CH}_{2}\right), 2.69-2.64\left(\mathrm{~m}, 8 \mathrm{H}, \mathrm{CH}_{2}\right), 2.21$ (s, 6H, $\left.\mathrm{CH}_{3}\right) \cdot{ }^{13} \mathrm{C}$ NMR $\left(100 \mathrm{MHz}, \mathrm{CDCl}_{3}, \mathrm{TMS}\right): \delta=$ $168.55,163.29,160.81,136.66,134.98,133.65$, $129.28,125.44,123.70,114.08,61.59,53.78,52.85$, 52.24,18.6. IR (KBr): $\delta=1655(\mathrm{C}=0)$. LCMS m/z: cal. 389.17; found $390.70(M+1)$.
N-(2,6-dimethylphenyl)-2-(4-(2-fluorobenzyl) piperazin-1-yl)acetamide $8 \mathrm{e}$

Colour: white solid, Yield: 93, m.p: 162$163^{\circ} \mathrm{C} .{ }^{1} \mathrm{H}$ NMR $\left(400 \mathrm{MHz}, \mathrm{CDCl}_{3}, \mathrm{TMS}\right): \delta=8.65$ (s, $1 \mathrm{H}, \mathrm{NH}), 7.39-7.35(\mathrm{~m}, 1 \mathrm{H}, \mathrm{Ar}-\mathrm{H}), 7.24-7.23$ (d, J = 2Hz, 1H, Ar-H), 7.13-7.00 (m, 5H, Ar-H), 3.61 (s, $\left.2 \mathrm{H}, \mathrm{CH}_{2}\right), 3.18\left(\mathrm{~s}, 2 \mathrm{H}, \mathrm{CH}_{2}\right), 2.72\left(\mathrm{~s}, 4 \mathrm{H}, \mathrm{CH}_{2}\right)$, $2.58\left(\mathrm{~s}, 4 \mathrm{H}, \mathrm{CH}_{2}\right), 2.20\left(\mathrm{~s}, 6 \mathrm{H}, \mathrm{CH}_{3}\right) \cdot{ }^{13} \mathrm{C}$ NMR $(100$ $\left.\mathrm{MHz}, \mathrm{CDCl}_{3}, \mathrm{TMS}\right): \delta=168.51,162.16,160.16$, $134.97,133.62,131.53,128.97,128.89,127.15$, $124.15,124.35,115.40,61.63,55.03,52.90,18.62$. IR $(\mathrm{KBr}): \delta=1635(\mathrm{C}=0)$. LCMS m/z: cal. 355.21; found $356.71(M+1)$.

\section{Biological Screening \\ Antibacterial activity}

Compounds 6a-e and 8a-e were evaluated for antibacterial activity. Agar well diffusion method was adopted for antibacterial activity using known literature procedure ${ }^{45}$. Experiment was performed in triplicate the mean values of zone of inhibition were given in Table 1.

\section{Antifungal Activity}

Antifungal activities of all piperazine derivatives towards two mold fungi were studied, viz. Candida albicans (human pathogen) Aspergillus flavus (mold). Poisoned food technique method was used to assess the antifungal activity of the synthesized compounds ${ }^{46}$, Nystatin $(10 \mu \mathrm{g} / \mathrm{disc})$ was used as standard fungicide. The experiments were performed in triplicate, average values were reported in Table 2.

\section{Anthelmintic activity}

Anthelmintic activity of compounds 6a-e and $8 \mathrm{a}$-e were evaluated using Pheretima posthuma (Indian Earthworm), anthelmintic activity of the compounds were evaluated as per the standard experimental method reported ${ }^{47}$. Time taken for paralysis and time taken for death were documented in minutes and the observed results were given in Table 3 .

\section{Molecular docking}

\section{Pharmacokinetic properties prediction}

The drug-specificity of the compounds was determined in accordance with Lipinski's rule of five ${ }^{48-49}$. Newly synthesized compounds were subject to the QICPROP50 computation software. Schrodinger software modules are designed to detect pharmacokinetic properties such as absorption, delivery, metabolism and division (ADME). The 
ligands for the installation were developed using LigPrep, Schrödinger. Energy was minimized by the OPLS 2005 force field ${ }^{51}$. Protein was prepared using Maestro's protein preparation wizard ${ }^{52}$. Docking was performed using GLIDE, Schrödinger. The GLIDE XP (extra nozzle) method was used for installation calculations ${ }^{53}$.

\section{RESULTS AND DISCUSSION}

\section{Chemistry}

The basic acetamide piperazine skeleton i.e. $\mathrm{N}$-(2,6-dimethylphenyl)-2-(piperazin-1-yl)acetamide 4 was synthesized as per the reported literature ${ }^{54}$ and confirmed by IR, ${ }^{1} \mathrm{H}$ and ${ }^{13} \mathrm{C}$ NMR. Scaffold $\mathrm{N}$-(2,6- dimethylphenyl)-2-(piperazin-1-yl) acetamide 4 on reaction with different substituted benzene sulfonyl chlorides $5 a-e$ gave pure piperazine sulfonamides 6a-e and with substituted benzyl chlorides 7a-e gave alkylated piperazine derivatives $8 \mathrm{a}-\mathrm{e}$ in good yield. Proton NMR of the title compounds $6 a-e$ were characterised by their resonance of aromatic protons in the range of 7.0-7.6 ppm, aliphatic protons at 2.1-3.2 ppm and $\mathrm{NH}$ proton of amide group resonate at chemical shift of $8.2 \mathrm{ppm}$. In case of 8a-e series, aromatic protons appear in the range of 6.9-7.4 ppm, aliphatic protons resonate in between 2.2-3.7 ppm. Interestingly $\mathrm{NH}$ proton of amide series shifted to downfield at $8.6 \mathrm{ppm}$ due to electron withdrawing nature of halogen substituents in the phenyl ring. In Carbon- ${ }^{13} \mathrm{CNMR}$, characteristic carbonyl carbon of 6a-e and 8a-e resonate at 167 and $168 \mathrm{ppm}$ respectively. Aromatic as well as aliphatic carbons of both the series appeared in the range 115$135 \mathrm{ppm}$ and 50-60 ppm respectively, mass spectra of all the compounds are in good agreement.

\section{Biological Screening \\ Antibacterial Activity}

Compounds 6a-e and 8a-e showed significant inhibitory action against tested Escherichia coli and Staphylococcus aureus strains. Initially different concentrations of $5 \mu \mathrm{g}, 15 \mu \mathrm{g}, 25 \mu \mathrm{g}$, $35 \mu \mathrm{g}$ and $50 \mu \mathrm{g} / \mathrm{mL}$ have been tried. At $50 \mu \mathrm{g} /$ $\mathrm{mL}$, no bacteria growth was observed hence this concentration was considered as minimum inhibitory concentration (MIC). Data obtained are presented in Table 1, shows that the derivatives are active at and above $50 \mu \mathrm{g} / 50 \mathrm{~mL}$. Compounds 8b, 8c, $6 \mathrm{e}$ and $6 \mathrm{c}$ displayed highest activity against $E$. coli and Staphylococcus aureus compared to Ciprofloxacin.

Table 1: Antibacterial activity Zone of Inhibition ( $\mathrm{mm})$ of 6a-e and 8a-e

\begin{tabular}{cccc}
\hline Samples & $\begin{array}{c}\text { Treatment } \\
(\mu \mathrm{g} / \mathrm{ml})\end{array}$ & $\begin{array}{c}\text { Escherichia coli } \\
(\text { Mean } \pm \text { SE) }\end{array}$ & $\begin{array}{c}\text { Staphylococcus aureus } \\
(\text { Mean } \pm \text { SE })\end{array}$ \\
\hline 6a & 50 & $15.44 \pm 0.55$ & $6.43 \pm 0.30$ \\
$6 \mathrm{~b}$ & 50 & $16.01 \pm 0.34$ & $7.14 \pm 0.33$ \\
$6 \mathrm{c}$ & 50 & $17.62 \pm 0.62$ & $8.41 \pm 0.15$ \\
$6 \mathrm{~d}$ & 50 & $16.12 \pm 0.27$ & $7.06 \pm 0.41$ \\
$6 \mathrm{e}$ & 50 & $17.91 \pm 0.24$ & $8.89 \pm 0.42$ \\
$8 \mathrm{a}$ & 50 & $12.92 \pm 0.30$ & $12.98 \pm 0.16$ \\
$8 \mathrm{~b}$ & 50 & $26.89 \pm 0.23$ & $13.35 \pm 0.20$ \\
$8 \mathrm{c}$ & 50 & $25.45 \pm 0.37$ & $12.96 \pm 0.16$ \\
$8 \mathrm{~d}$ & 50 & $15.93 \pm 0.76$ & $12.21 \pm 0.25$ \\
$8 \mathrm{e}$ & 50 & $14.10 \pm 0.41$ & $11.80 \pm 0.29$ \\
Ciprofloxacin & 10 & $31.00 \pm 0.43$ & $15.00 \pm 0.39$ \\
\hline
\end{tabular}

\section{Antifungal activity}

Compounds 6a-e and 8a-e showed more or less inhibitory action against Candida albicans and Aspergillus flavus as compared to standard Nystatin. Initially different concentrations of $5 \mu \mathrm{g}, 15 \mu \mathrm{g}, 25 \mu \mathrm{g}$, $35 \mu \mathrm{g}$ and $50 \mu \mathrm{g} / \mathrm{mL}$ have been tested. At $50 \mu \mathrm{g} / \mathrm{mL}$ was considered as minimum inhibitory concentration (MIC) for both the fungal strains.

Table 2: Antifungal activity Zone of Inhibition ( $\mathrm{mm}$ ) of 6a-e and 8a-e

\begin{tabular}{cccc}
\hline Samples & $\begin{array}{c}\text { Treatment } \\
(\mu \mathrm{g} / \mathrm{ml})\end{array}$ & $\begin{array}{c}\text { Candida albicans } \\
(\text { Mean } \pm \text { SE) }\end{array}$ & $\begin{array}{c}\text { Aspergillus flavus } \\
(\text { Mean } \pm \text { SE })\end{array}$ \\
\hline $6 a$ & 50 & $10.75 \pm 0.24$ & $16.13 \pm 0.63$ \\
$6 \mathrm{~b}$ & 50 & $10.12 \pm 0.42$ & $17.56 \pm 0.52$ \\
$6 \mathrm{c}$ & 50 & $15.03 \pm 0.22$ & $12.87 \pm 0.13$ \\
$6 \mathrm{~d}$ & 50 & $15.74 \pm 0.11$ & $13.09 \pm 0.13$ \\
$6 \mathrm{e}$ & 50 & $11.05 \pm 0.17$ & $11.14 \pm 0.26$ \\
$8 \mathrm{a}$ & 50 & $23.25 \pm 0.17$ & $18.44 \pm 0.17$ \\
$8 \mathrm{~b}$ & 50 & $23.97 \pm 0.12$ & $19.09 \pm 0.43$ \\
$8 \mathrm{c}$ & 50 & $25.67 \pm 0.25$ & $19.12 \pm 0.26$ \\
$8 \mathrm{~d}$ & 50 & $14.10 \pm 0.62$ & $12.23 \pm 0.23$ \\
$8 \mathrm{e}$ & 50 & $25.52 \pm 0.29$ & $18.20 \pm 0.11$ \\
Nystatin & 10 & $29.33 \pm 0.13$ & $21.33 \pm 0.23$ \\
\hline
\end{tabular}

Data presented in Table 2 showed that the derivatives were active at and above $50 \mu \mathrm{g} / 50 \mu \mathrm{L}$. Amongst all, derivatives $8 \mathrm{c}, 8 \mathrm{e}, 8 \mathrm{~b}$ and $8 \mathrm{a}$ showed significant activity against Candida albicans and Aspergillus flavus. All other molecules showed moderate activity against tested microorganisms compared to standard Nystatin.

\section{Anthelmintic Activity}

Compounds 6a-e and 8a-e tested for 
anthelmintic activity against Pheretima posthuma (Indian earthworm) showed moderate results compared to standard piperazine citrate. Earthworms belonging to control group showed paralysis time of $142.33 \pm 0.49 \mathrm{~min}$ and death time of $167.17 \pm 0.87$ minutes. The paralysis and death time for test samples $6 a-e$ and $8 a-e$ at the concentrations of $50 \mathrm{mg} / \mathrm{mL}$ were reported in Table 3 . Standard piperazine citrate exhibited $25.17 \pm 0.48$ and $47.00 \pm 0.58 \mathrm{~min}$ time for paralysis and death at concentration of $50 \mathrm{mg} /$ $\mathrm{mL}$. Examination of anthelmintic activity revealed that compound 8d, 6a and 6e showed significant activity against Pheretima posthuma.

Table 3: Anthelmintic activity of compounds 6a-e and 8a-e

\begin{tabular}{cccc}
\hline Test Samples & $\begin{array}{c}\text { Concentration } \\
(\mathrm{mg} / \mathrm{mL})\end{array}$ & $\begin{array}{c}\text { Time taken for } \\
\text { paralysis }(\mathrm{min})\end{array}$ & $\begin{array}{c}\text { Time taken } \\
\text { for death(min) }\end{array}$ \\
\hline Control & - & $142.33 \pm 0.49$ & $167.17 \pm 0.87$ \\
& & & \\
6a & 50 & $28.01 \pm 0.86$ & $28.07 \pm 1.16$ \\
$6 \mathrm{~b}$ & 50 & $29.27 \pm 0.60$ & $29.34 \pm 1.03$ \\
$6 \mathrm{c}$ & 50 & $35.81 \pm 1.26$ & $36.47 \pm 0.60$ \\
6d & 50 & $29.00 \pm 1.05$ & $29.27 \pm 0.95$ \\
6e & 50 & $28.00 \pm 1.06$ & $28.41 \pm 0.27$ \\
8a & 50 & $30.23 \pm 1.48$ & $30.43 \pm 0.87$ \\
8b & 50 & $36.31 \pm 0.67$ & $37.33 \pm 1.33$ \\
8c & 50 & $37.97 \pm 0.48$ & $39.89 \pm 0.76$ \\
8d & 50 & $28.31 \pm 1.26$ & $31.27 \pm 0.48$ \\
8e & 50 & $30.34 \pm 1.06$ & $37.97 \pm 0.58$ \\
Piperazine citrate & 50 & $25.17 \pm 0.48$ & $47.00 \pm 0.58$ \\
\hline
\end{tabular}

\section{Molecular Docking}

\section{Prediction of Pharmacokinetic Properties}

The 2D structures of compounds 6a-e and $8 \mathrm{a}-\mathrm{e}$ were subjected to a computational program using Qikprop module of Schrödinger software for the in silico determination of pharmacokinetic properties. The statistical parameters of the pharmacokinetic properties of compounds were tabulated in Table 4.

The pharmacokinetic properties predictions of compounds 6a-e and 8a-e indicate that all the compounds were endowed with drug like properties. The results revealed that there is no violation in agreement with the rule of five. The molecular weight of compounds ranges from 355 to 466 a.m.u. The number of hydrogen bond donor is one whereas the hydrogen bond acceptor values vary from 6.5 to 10 . In addition, the partition coefficient values of all compounds are less than five.
Table 4. Lipinski's rule of five factors of compounds 6a-e and 8a-e

\begin{tabular}{cccccc}
\hline \multicolumn{5}{c}{ Factors of Lipinski's rule of five } \\
Comp. & $\begin{array}{c}\text { mol_MW } \\
(<500)\end{array}$ & $\begin{array}{c}\text { Donor HB } \\
(<5)\end{array}$ & $\begin{array}{c}\text { Accpt HB } \\
(<10)\end{array}$ & $\begin{array}{c}\text { QPlogPo/w Rule of Five } \\
(<5)\end{array}$ \\
\hline $6 \mathrm{a}$ & 417.522 & 1 & 9.75 & 1.536 & 0 \\
$6 \mathrm{~b}$ & 421.941 & 1 & 9 & 1.778 & 0 \\
$6 \mathrm{c}$ & 432.493 & 1 & 10 & 0.847 & 0 \\
$6 \mathrm{~d}$ & 466.392 & 1 & 9 & 1.855 & 0 \\
$6 \mathrm{e}$ & 401.523 & 1 & 9 & 1.646 & 0 \\
$8 \mathrm{a}$ & 416.36 & 1 & 6.5 & 3.026 & 0 \\
$8 \mathrm{~b}$ & 355.454 & 1 & 6.5 & 2.694 & 0 \\
$8 \mathrm{c}$ & 406.354 & 1 & 6.5 & 3.525 & 0 \\
$8 \mathrm{~d}$ & 389.899 & 1 & 6.5 & 3.175 & 0 \\
$8 \mathrm{e}$ & 355.454 & 1 & 6.5 & 2.804 & 0 \\
\hline
\end{tabular}

The tested compounds 8 a-e have more than 82 percentage of human oral absorption whereas 6a-e have more than 74 percentage of human oral absorption except compound $6 \mathrm{c}$ which has less than $60 \%$. The aqueous solubility (QPlogS) parameter and $\mathrm{IC}_{50}$ values of HERG $\mathrm{K}+$ channel blockage (QPlogHERG) of all the tested compounds possess permissible parameters.

\section{Docking Studies}

The molecular docking studies were carried out using Lead IT for antibacterial, antifungal and anthelmintic activity. Docking energy of the synthesized molecules along with standards was tabulated in Table 5. The Molecular Docking result shows that compounds 8a-e have higher docking energy compared with antibacterial standard ciprofloxacin. Antifungal standard Nystatin has the highest docking score compared to all the compounds, but in case of anthelmintic activity most of the compounds have docking score which are more comparable to piperazine citrate. All the molecules were docked in the active sites of respective proteins with effectively. The fitting of the most potent compounds $8 \mathrm{~b}, 8 \mathrm{c}, 6 \mathrm{c}$ and $6 \mathrm{e}$ against bacteria, $8 \mathrm{a}, 8 \mathrm{~b}, 8 \mathrm{c}$ and $8 \mathrm{e}$ against fungi, the anthelmintic activity of $6 \mathrm{c}, 8 \mathrm{~b}$ and $8 \mathrm{c}$ are discussed below. Since conventional hydrogen bonding is important, our molecular docking discussion is restricted to hydrogen bonding even though compounds show interactions like hydrophobic, $\rho$ i- $\rho \mathrm{i}$ stacked $\rho$ i-alkyl interactions. 
Table 5: Docking energy in Kcal/mole of Target molecules

\begin{tabular}{cccc}
\hline Comp. & $\begin{array}{c}\text { Antibacterial } \\
\text { PDB ID:3ACX }\end{array}$ & $\begin{array}{c}\text { Antifungal } \\
\text { PDBID:1IYK }\end{array}$ & $\begin{array}{c}\text { Anthelmintic } \\
\text { PDBID:1SAO }\end{array}$ \\
\hline 6a & -6.115 & -5.658 & -12.873 \\
$6 \mathrm{~b}$ & -7.212 & -5.458 & -7.149 \\
$6 \mathrm{c}$ & -11.532 & -7.093 & -7.139 \\
$6 \mathrm{~d}$ & -7.176 & -7.755 & -7.257 \\
$6 \mathrm{e}$ & -10.773 & -5.545 & -12.873 \\
8a & -7.921 & -18.706 & -7.283 \\
8b & -12.121 & -16.845 & -7.811 \\
8c & -11.463 & -19.471 & -8.332 \\
8d & -7.248 & -7.939 & -13.225 \\
8e & -8.511 & -17.664 & -7.969 \\
Ciprofloxacin & -9.185 & - & - \\
Nystatin & - & -23.499 & - \\
Piperizine Citrate & - & - & -9.254 \\
\hline
\end{tabular}

Binding mode of potent Antibacterial compounds

In binding pose of nitro substituted piperazine sulfonamide $6 \mathrm{c}$, the prominent interactions observed are as follows one hydrogen bond formed by donating hydrogen from $\mathrm{NH}$ group to OD1 group of Asp48 residue. The amino acid residues Asn168, Arg171 and Tyr248 forms common hydrogen interaction with $\mathrm{O} 21$ group of methyl piperazine sulfonamide 6e. 011 atom of Alkylated piperazine derivative $8 \mathrm{~b}$ have hydrogen bond interaction with Amino acid residues Arg171 and Tyr248. There are each one hydrogen bond established between Asp48 amino acid and compounds 8c. The docking pose of the proteins and ligands are shown in Figure1.

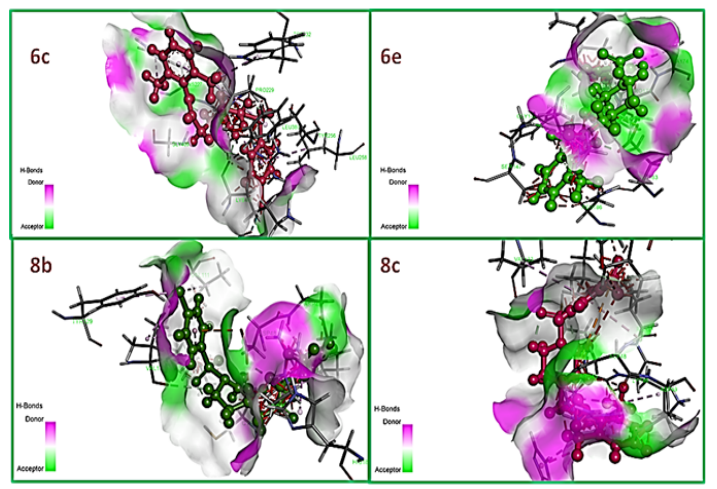

Fig. 1. Docking poses of compounds $6 c, 6 e, 8 b$ and $8 c$ with protein $3 \mathrm{ACX}$

Binding mode of potent Antifungal compounds Molecular docking of the compounds into the active site of $\mathrm{N}$-myristoyltranferase shown in Fig. 2 reveals an 010 group of His227 forms one conventional hydrogen bond with bromo and fluro substituents separately in the compounds $8 \mathrm{a}$ and $8 \mathrm{~b}$ respectively. OD1 group of Asp110 residue accepts hydrogen from $\mathrm{NH}$ group of compound $8 \mathrm{c}$ to form a conventional hydrogen bond. Compound $8 \mathrm{e}$ donate hydrogen bond to Asp412 residue. These are the main significant interactions observed in the active pocket of antifungal protein and our molecules.

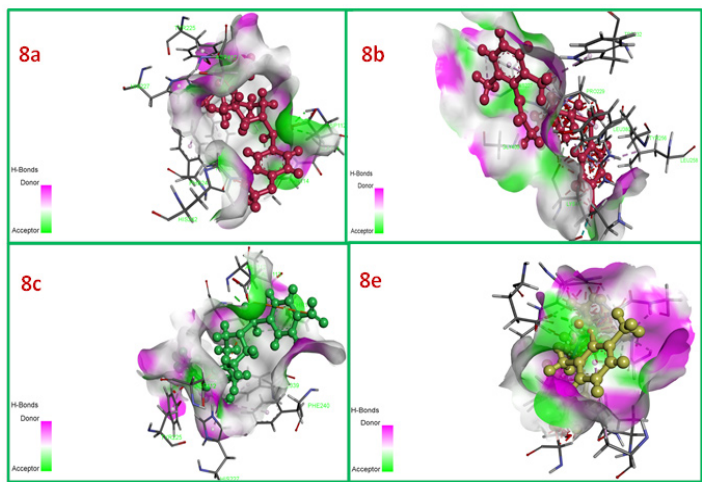

Fig. 2. Binding pose of the active compound's $8 a, 8 b, 8 c$ and 8e with Protein 1IYK

Binding mode of potent Anthelmintic compounds

In the binding pocket of the protein tubulin shows two hydrogen bonding interaction between $\mathrm{O} 20, \mathrm{O} 21$ groups of piperazine sulfonamide $6 \mathrm{c}$ and $\mathrm{NH}, \mathrm{HE} 22$ groups of GIn11. Compound 8b with fluro and chloro substituents forms three hydrogen bond interaction with Phe141, Gly146 and Glu183. Three hydrogen interactions were observed between alkylated piperazine derivative 8c with Phe141, Gly146 and Glu183 as shown in Fig. 3. These interactions may responsible for the high docking score of the active compounds.

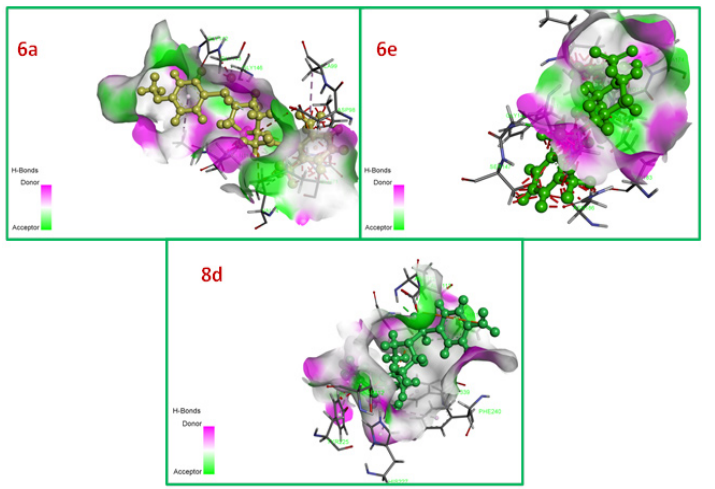

Fig. 3. Binding pose of the active compound's $6 a, 6 e$ and $8 d$ with Protein 1SA0

Finger print Analysis

In recent years, many fluorescent compounds 
have been used as a labeling material for fingerprinting in forensics ${ }^{55}$. Usually, LFGs are often not seen or investigated in places of crime. Extraordinary fluorescent powders were used to improve LFG in crime scene, and have been well-known for the last few decades ${ }^{56}$. The powder dusting method is the most widely used method for the development of LFPs in forensic science. ${ }^{57,58}$ However, these powders were effective in detecting LFPs under normal conditions. Powder-dusting method using these powders is still challenged by severe problems, such as low sensitivity, low contrast, high background interference, and high auto fluorescence interactions ${ }^{59-61}$.

\section{Revelation of eccrine LFPs using synthesized compounds}

All eccrine LFPs were collected by a donor using cleaned and dried hands. Later, the fingers were pressed at low pressure on various surfaces with infiltrating and non-infiltrating materials at $37^{\circ} \mathrm{C}$ temperature to obtain LFP. Each compound is carefully applied to the eccrine LFPs to improve the LFP and the excess compound powder was removed by light brushing method. The obtained fingerprints were photographed with normal white light with a digital camera Nikon D3100/AF_S Nikkor50 mm f/2.8G ED.

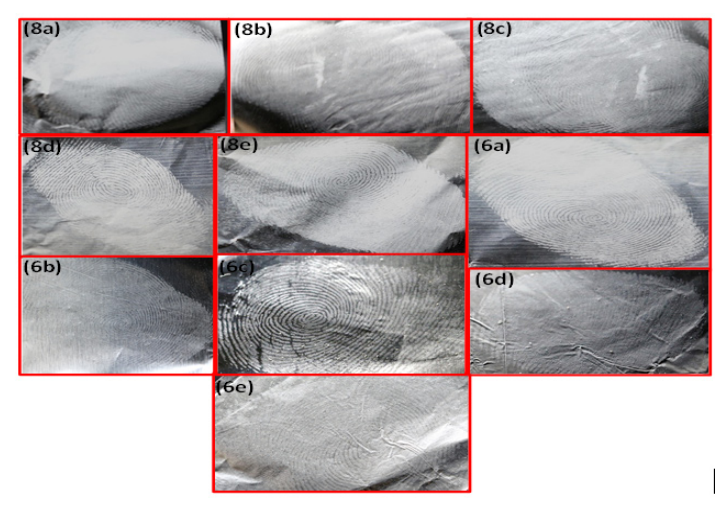

Fig. 4. Fingerprint visualization of $8 \mathrm{a}-\mathrm{e}$ and $6 \mathrm{a}-\mathrm{e}$ samples on aluminum foil surface under normal light illumination

Figure 4 shows the development of eccrine LFPs of synthesized compounds on aluminum foil surface to examine their labeling efficacy in forensic applications. This visualization of eccrine LFPs on aluminum foil surface was developed under normal white light illumination. From the Figure it is found that among the entire samples, sample $6 \mathrm{c}$ shows the clear ridge details after powder dusting method. Hence, sample $6 c$ was optimized and used as labeling agent on various porous and non-porous surfaces such as, magazine covers (porous), glass slide, spray bottle cap, compact disk, black plastic bottle, coin, granite, spoon and highlighter pen surface (Figure 5).

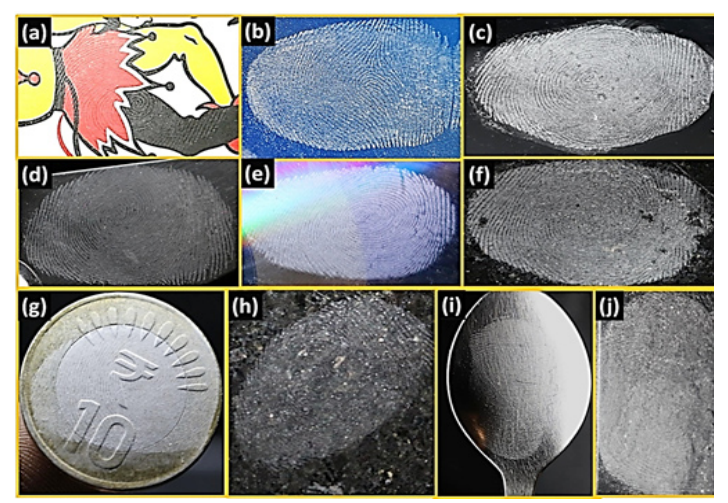

Fig. 5. Fingerprint visualization of $6 c$ optimized on $(a, b)$ magazine cover, (c) glass slide, (d) spray bottle cap, (e) compact disk, (f) black plastic bottle, (g) coin, (h) granite, (i) spoon and (j) highlighter pen surface.

From the images it is clear that Minutiae ridges have been detected in all surfaces without any color barrier, indicating that the improvement of eccrine finger prints by powder dusting method with compound $6 \mathrm{c}$ used as labeling agents on forensic relevant porous and non-porous surfaces.

\section{CONCLUSION}

In this study two new series of compounds were synthesized and evaluated for their antibacterial, antifungal, anthelmintic activity. Amongst all the tested compounds $6 \mathrm{c}, 6 \mathrm{e}, 8 \mathrm{~b}$ and $8 \mathrm{c}$ showed noteworthy antibacterial activity against $E$. coli and Staphylococcus aureus. Compounds 8a, 8b, $8 \mathrm{c}$ and $8 \mathrm{e}$ showed significant antifungal activity and other alkylated piperazine derivatives $6 a, 6 e$ and $8 \mathrm{~d}$ showed good anthelmintic activity. It can be concluded that acetamide derivatives linked to piperazine show considerable antibacterial, antifungal, and anthelmintic activity. Additionally, the molecular docking and drug-likeliness shows that these compounds have good docking energies. This result implies that some of these compounds are new potent antimicrobial and anthelmintic agents. Compound $6 \mathrm{c}$, which has been tested for latent fingerprints detection, has shown that fingerprints are better than other substances and may be used for forensic application. 


\section{ACKNOWLEDGEMENT}

The authors are grateful to Tumkur University and College of Science for the laboratory facilities and also authors acknowledge Annamalai
University for providing docking software facility.

\section{Conflict of Interest}

Authors declare no conflict of interest.

\section{REFERENCES}

1. Shaw, J. E.; Sicre, R. A.; Zimmet, P. Z Diabetes Res. Clin. Pract., 2010, 87, 4-14.

2. Zhang, P. X.; Zhang, J.; Brown, D.; Vistisen.; Sicree, R.; Shaw, J.; Nichols, G. Diabetes Res. Clin. Pract., 2010, 87, 293-301.

3. Alberti, G.; Zimmet, P.; Shaw, J. E.; Bloomgarden, Z.; Kaufman, F.; Silink, M. Diabetes Care., 2004, 27, 1798-1811.

4. Fowler, M. J. Clin. Diabete., 2008, 26, 77-82.

5. Yonemoto, R.; Shimada, M.; Puteri, M. D. P.T.; Kato, E.; Kawabata, J. J. Agric. Food Chem., 2014, 62, 8411-8414.

6. Ahmed, A.; Molvi, K. I.; Nazim, S.; Baig, I.; Memon, T.; Rahil, M. J. Chem. Pharm. Res., 2012, 4, 872-880.

7. Akkoc, M. K.; Yuksel, M.Y.; Durmaz, I.; Atalay, R. C. Turk. J. Chem., 2012, 36, 515-525.

8. Meher, C. P.; Rao, A. M.; Omar, M. Asian J. Pharm. Sci. Res., 2013, 3, 43-60.

9. Jain, V. K.; Jain, B.; Sharma, U. K.; Saha, D. Int. J. Curr. Pharm. Res., 2011, 3, 66-70.

10. Joshi, N. K.; Kundariya, D. S.; Parmar, J. M. Int. J. Chem. Tech. Res., 2012, 24, 1503-1508.

11. Gan, L. L.; Fang, B.; Zhou, C. H. Bull. Korean Chem., 2010, 31, 3864-3692.

12. Mukherjee, D.; Mukhopadhayay, A.; Shridhara Bhat, K.; Shridhara, A. M.; Rao, K. S. Int. J. Pharm. Pharm. Sci., 2014, 6, 567-571.

13. Ibezim, P.R.; Duchowicz, E.;Ortiz, P.R. E.V.; Castro, E. A. Chemometr. Intell. Lab., 2012, 110, 81-88.

14. Cho, S. D.; Song, S. Y.; Kim, K. H.; Zhao, B. X.; Ahn, C.; Joo, W. H.; Yoon, Y. J.; Falck, J. R.; Shin, D. S. Bull. Korean Chem. Soc., 2004, 25, 415 - 416.

15. Ashour, S.; Kattan, N. J. Pharm. Anal., 2012, 2, 437- 442.

16. Al-Shaalan, N. H. Saudi Pharm. J., 2012, 20, 255-262.

17. Kalai, T.; Khan, M.; Balog, M.; Kutala, V. K.; Kuppusamy, P.; Hideg, K. Bioorg. Med. Chem., 2006, 14, 5510-5516.

18. Ignat, A.; Zahari, V.; Mogos, C.; Palibroda, N.; Cristea, C.; Dumitrescu, L. S. Farmacia., 2010, 3, 290-302.
19. Tyagi, S.; Kumar, S.; Kumar, A.; Singla, M. IJPWR., 2010, 22, 1-16.

20. Finch, R. A.; Shyam, K.; Penketh, P.G.; Sartorelli, A. C. Cancer Res., 2001, 61, 3033-3038.

21. Silva, L. L.; Oliveira, K. N.; Nunes, R. J. ARKIVOC., 2006, 13, 124-129.

22. Tyagi, S.; Kumar, S.; Kumar, A.; Singla, M. IJPWR., 2010, 22, 1-16.

23. Hulme, C.; Cherrier, M. P.; Tetrahedron Lett., 1999, 40, 5295.

24. Hansch, C.; Sammes, P. G.; Taylor, J. B. Compr. Med. Chem., 1990, 2.

25. Kanda, Y.; Kawanishi, Y.; Oda, K.; Sakata, T.; Mihara, S.; Asakura, K.; Kanemasa, T.; Ninomiya, M.; Fujimoto, M.; Kanoike, T. Bioorg. \& Med. Chem., 2001, 9, 897-904.

26. Stokes, S. S.; Albert, R.; Buurman Ed, T.; Andrews, B.; Shapiro, A. B.; Green, O. M.; McKenzie, A. R.; Otterbein, L. Bioorg. \& Med. Chem. Lett., 2012, 22, 7019.

27. Chibale, K.; Haupt, H.; Kendrick, H.; Yardley, V.; Saravanamuthu, A.; Fairlamb, A. H.; Croft, S. L. Bioorg \& Med. Chem. Lett., 2001, 11, 2655.

28. Ezabadi, I.; Camoutsis, R. C.; Zoumpoulakis, P.; Geronikaki, A.; Sokovic, M.; Glamocilija, J. C. Bioorg \& Med. Chem., 2008, 16, 1150.

29. Kennedy, J. F.; Thorley, M.; Kleeman, A.; Engel, J.; Kutscher, B.; Reichert, D. Thieme Stuttgart. $3^{\text {rd }}$ Ed., $1999,7$.

30. Serradeil-Le Gal, C. Cardiovascular Drug Rev., 2001, 19, 201.

31. Natarajan, A.; Guo, Y.; Harbinski, F.; Fan, Y. H; Chen, H.; Luus, L.; Diercks, J.; Aktas, H.; Chorev, M.; Halperin J. A. J. Med. Chem., 2004, 47, 4979.

32. Vullo, D.; De Luca, V.; Scozzafava, A.; Carginale, V.; Rossi M.; Supuran, C.T.; Capasso, C. Bioorg. \& Med. Chem., 2013, 21, 4521.

33. Autore, G.; Caruso, A.; Marzocco, S.; Nicolaus, B.; Palladino, C.; Pinto, A. ; Popolo, A.; Sinicropi, M. S.; Tommonaro G.; Saturnino, C. Molecules., 2010, 15, 2028.

34. Ayhan-Kılcıgil, G.; Gurkan, S.; Coban, T.; Ozdamar, E. D.; Can-Eke, B. Chem. Biol. Drug. Des., 2012, 79, 869. 
35. Jawed, H.; Shah, S. U. A.; Jamall, S.; Simjee, S. U. Int. Immuno pharmacol., 2010, 10, 900.

36. Kanagarajan, V.; Thanusu, J.; Gopalakrishnan, M. Eur. J. Med. Chem., 2010, 45, 1583.

37. Sawant, R.; Kawade, D. Acta Pharmaceutica. 2011, 61, 353.

38. Zhan, P.; Liu, X.; Li, Z.; Fang, Z.; Li, Z.;Wang, D.; Pannecouque, C.; Clercq, E. D. Bioorg. Med. Chem., 2009, 17, 5920-5927.

39. Chan, H. C.; Kuo, S.C.; Huang, L.-J.; Liu, C.-H.; Hsu, S.-L., 2013, 467, 31-39.

40. Kılcıgil, G.A.; Gurkan, S.; Coban, T.; Ozdamar, E. D.; Can-Eke, B. Chem. Biol. Drug. Des., 2012, 79, 869.

41. Duckworth, C. A. US patent. 5, 538, 938,199.

42. Ikeuchi, T.; Ohkawa, T.; Ohno, S. EP patent., 2008.

43. Zablocki, J. A.; Prabha, N.; Ibrahim, Shenk, K.; Elzein, E.; Palle, V.; US patent. 20050272745 A1.

44. a) Wandler, A. G.; Fr. Patent. 1, 334944, 1963; b) Schmutz, H. U.S. Patent. 3539, 573, 1970.

45. Seeley, H. W.; Van Denmark, P. J. Laboratory manual of Microbiology. $2^{\text {nd }}$ Ed. Bombay, Taraporewala D. B. Sons and Co, Microbes in action., 1975, 55-80.

46. Balouiri, M.; Sadiki, M., Ibnsouda, S. K. J. Pharma. Anal., 2016, 6, 71-79.

47. Satish, B.; Kosalge.; Ravindra, A.; Fursule. Asian J. Pharma. Clin. Res., 2009, 2, 69-71.

48. Lipinski, C. A.; Lombardo, F.; Dominy, B. W.; Feency, P. J. Adv. Drug Deliv. Rev., 1997, 23, 3.

49. Lipinski, C. A.; Lombardo, F.; Dominy, B. W.; Feency, P.; J. Adv .Drug Deliv. Rev., 2001, 46, 3.

50. QikProp, version 3.5, Schrödinger, LLC, New York, NY., 2014.
51. LigPrep version 3.1, Schrödinger, LLC, New York, NY., 2014.

52. Maestro version 9.8, Schrödinger, LLC, New York, NY., 2014.

53. Glide, version 3.3, Schrödinger, LLC, New York, NY., 2014.

54. Michel, G.; Jef, C.; Ivan, V.; Dirk, D. S.; Stef, L. Organic Process Research \& Development., 2003, 7, 939-941.

55. Basavaraj, R.B.; Nagabhushana, H.; Darshan, G.P.; Daruka Prasad, B.; Sharma, S. C.; Venkatachalaiah, K.N. J. Ind. Eng. Chem., 2017, 51, 90-105.

56. Basavaraj, R.B.; Nagabhushana, H.; Darshan, G.P.; Daruka Prasad, B.; Rahul, M.; Sharma, S.C.; Sudaramani, R.; Archana, K.V. Dyes Pig., 2017, 147, 364-377.

57. Dhanalakshmi, M.; Nagabhushana, H.;Basavaraj, R.B.; Prabhukumar, G. D.; Prasad, D. B. ACS Sustainable Chem. Eng., 2018, 6, 5214-5226.

58. Shivaraja, G.; Sreenivasa, S.; Ramesha, A. R.; Madhu Chakrapani, T. R.; Nagabhushana, H. Chemistry Select., 2018, 3, 8111 - 8117.

59. Deepthi, N.H.; Darshan, G.P.; Basavaraj, R.B.; Daruka Prasad, B.; Nagabhushana, H. Actuators. B., 2018, 255, 3127-3147.

60. Suresh, C.; Nagabhushana, H.; Basavaraj, R.B.; Darshan, G.P.; Kavyashree, D.; Daruka Prasad, B.; Sharma, S.C.; Vanithamani, R. J. Colloid and Interface Sci., 2018, 518, 200-215.

61. Venkatachalaiah, K.N.; Nagabhushana, H.; Darshan, G.P.; Basavaraj, R.B.; Daruka Prasad, B. Sens. Actuators., 2017, B251, 310-325. 